\title{
On the Law of Large Numbers for Nonmeasurable Identically Distributed Random Variables
}

by

\author{
Alexander R. PRUSS \\ Presented by Stanisław KWAPIEN
}

\begin{abstract}
Summary. Let $\Omega$ be a countable infinite product $\Omega_{1}^{\mathbb{N}}$ of copies of the same probability space $\Omega_{1}$, and let $\left\{\Xi_{n}\right\}$ be the sequence of the coordinate projection functions from $\Omega$ to $\Omega_{1}$. Let $\Psi$ be a possibly nonmeasurable function from $\Omega_{1}$ to $\mathbb{R}$, and let $X_{n}(\omega)=$ $\Psi\left(\Xi_{n}(\omega)\right)$. Then we can think of $\left\{X_{n}\right\}$ as a sequence of independent but possibly nonmeasurable random variables on $\Omega$. Let $S_{n}=X_{1}+\cdots+X_{n}$. By the ordinary Strong Law of Large Numbers, we almost surely have $E_{*}\left[X_{1}\right] \leq \liminf S_{n} / n \leq \lim \sup S_{n} / n \leq E^{*}\left[X_{1}\right]$, where $E_{*}$ and $E^{*}$ are the lower and upper expectations. We ask if anything more precise can be said about the limit points of $S_{n} / n$ in the nontrivial case where $E_{*}\left[X_{1}\right]<E^{*}\left[X_{1}\right]$, and obtain several negative answers. For instance, the set of points of $\Omega$ where $S_{n} / n$ converges is maximally nonmeasurable: it has inner measure zero and outer measure one.
\end{abstract}

1. Introduction. Ordinary random variables are $P$-measurable functions on a probability space $(\Omega, \mathcal{F}, P)$, where $\mathcal{F}$ is a $\sigma$-field on $\Omega$. By the ordinary Strong and Weak Laws of Large Numbers, if $X_{1}, X_{2}, \ldots$ are measurable identically distributed random variables with finite expectation, then $\left(X_{1}+\cdots+X_{n}\right) / n \rightarrow E\left[X_{1}\right]$ almost surely (Strong Law) and in probability (Weak Law). But we can also ask what happens to long-term means of samples when the random variables are not measurable.

Let $(\Omega, \mathcal{F}, P)$ be a probability space. The following collects some known facts (see, e.g., [6, Lemmas 1.2.2 and 1.2.3]) that allow us to apply probabilistic techniques in the case of nonmeasurable random variables.

Proposition 1.1. Let $H$ be any subset of $\Omega$. Then there are measurable sets $H_{*}$ and $H^{*}$ such that $H_{*} \subseteq H \subseteq H^{*}$ and such that for any measurable

2010 Mathematics Subject Classification: 60F10, 60F05, 28A12.

Key words and phrases: law of large numbers, measurability, nonmeasurable random variables, probability. 
$A \subseteq H$ we have $P(A) \leq P\left(H_{*}\right)$ and for any measurable $B \supseteq H$ we have $P(B) \geq P\left(H^{*}\right)$. The sets $H_{*}$ and $H^{*}$ are uniquely defined up to sets of measure zero.

For any real-valued function $f$ on $\Omega$, there are measurable functions $f_{*}$ and $f^{*}$ such that $f_{*} \leq f \leq f^{*}$ everywhere and, for any measurable $g$ on $\Omega$ such that $g \leq f$ everywhere, we have $g \leq f_{*}$ almost surely, while for any measurable $h$ on $\Omega$ such that $f \leq h$ everywhere, we have $h \geq f^{*}$ almost surely. The functions $f_{*}$ and $f^{*}$ are uniquely defined up to almost sure equality.

The functions $f_{*}$ and $f^{*}$ are the maximal measurable minorant and minimal measurable majorant of $f$, respectively.

We then have $P_{*}(H)=P\left(H_{*}\right)$ and $P^{*}(H)=P\left(H^{*}\right)$, where $P_{*}$ and $P^{*}$ are the inner and outer measures generated by $P$. Note that $H$ is measurable with respect to the completion of $P$ if and only if $P_{*}(H)=P^{*}(H)$, in which case it has that value as its measure with respect to the completion of $P$.

We say that a set is maximally nonmeasurable provided that $P_{*}(H)=0$ and $P^{*}(H)=1$. Such a set is one all of whose measurable subsets have null measure and all of whose measurable supersets have full measure.

As a replacement for the independence assumption in the case of ordinary random variables, take our probability space $(\Omega, \mathcal{F}, P)$ to be the product of the probability spaces $\left(\Omega_{n}, \mathcal{F}_{n}, P_{n}\right)$, and let our sequence of possibly nonmeasurable random variables be a sequence of functions $X_{1}, X_{2}, \ldots$ on $\Omega$ such that $X_{n}\left(\omega_{1}, \omega_{2}, \ldots\right)$ depends only on the value of $\omega_{n}$, so that there is a function $\Psi_{n}$ such that $X_{n}\left(\omega_{1}, \omega_{2}, \ldots\right)=\Psi_{n}\left(\omega_{n}\right)$. We will say that $X_{1}, X_{2}, \ldots$ is then a sequence of independent identically distributed possibly nonmeasurable random variables (iidpnmrvs) providing that all the probability spaces $\left(\Omega_{n}, \mathcal{F}_{n}, P_{n}\right)$ are the same space $\left(\Omega_{1}, \mathcal{F}_{1}, P_{1}\right)$ and that $\Psi_{n}$ is the same function $\Psi$ for all $n$.

The following fact about product measures and the $(\cdot)_{*}$ and $(\cdot)^{*}$ operators follows from [6, Lemma 1.2.5].

Proposition 1.2. Suppose $(\Omega, \mathcal{F}, P)$ is the product of the probability spaces $\left(\Omega_{n}, \mathcal{F}_{n}, P_{n}\right)$ for $n=1,2, \ldots$ Let $\Psi_{n}$ be a function on $\Omega_{n}$. Let $X_{n}\left(\omega_{1}, \omega_{2}, \ldots\right)=\Psi_{n}\left(\omega_{n}\right)$. Let $Y_{n}\left(\omega_{1}, \omega_{2}, \ldots\right)=\left(\Psi_{n}\right)_{*}\left(\omega_{n}\right)$ and $Z_{n}\left(\omega_{1}, \omega_{2}, \ldots\right)$ $=\Psi_{n}^{*}\left(\omega_{n}\right)$. Then P-almost surely we have $\left(X_{n}\right)_{*}=Y_{n}$ and $X_{n}^{*}=Z_{n}$.

In particular, if $X_{1}, \ldots, X_{n}$ are iidpnmrvs, then $\left(X_{1}\right)_{*}, \ldots,\left(X_{n}\right)_{*}$ are identically distributed independent random variables, and so are $X_{1}^{*}, \ldots, X_{n}^{*}$. Let $S_{n}=X_{1}+\cdots+X_{n}$. From the Strong Law of Large Numbers as applied to $\left\{\left(X_{n}\right)_{*}\right\}$ and $\left\{X_{n}^{*}\right\}$ (and using the fact that if $\left|X_{1}\right|^{*}$ is integrable, then so are $\left(X_{1}\right)_{*}$ and $\left.X_{1}^{*}\right)$ it then follows that almost surely

$$
E\left[\left(X_{1}\right)_{*}\right] \leq \liminf _{n \rightarrow \infty} \frac{S_{n}}{n} \leq \limsup _{n \rightarrow \infty} \frac{S_{n}}{n} \leq E\left[X_{1}^{*}\right] .
$$


Here and elsewhere "almost surely" will mean except perhaps on a set of probability zero. Thus an event holds almost surely provided its lower probability is 1 . (In the case of complete measures, this is equivalent to the usual notion of holding almost surely as holding on a set of full measure.)

We can define the lower and upper expectations of $X_{1}$ as $E_{*}\left[X_{1}\right]=$ $E\left[\left(X_{1}\right)_{*}\right]$ and $E^{*}\left[X_{1}\right]=E\left[X_{1}^{*}\right]$, respectively (for more on lower and upper expectations, see [4]). Again, we have a trivial case when $E\left[\left(X_{1}\right)_{*}\right]=E\left[X_{1}^{*}\right]$ and then the Strong Law holds. In that case, $\left(X_{n}\right)_{*}=X_{n}^{*}$ almost surely (since $\left.\left(X_{n}\right)_{*} \leq X_{n} \leq X_{n}^{*}\right)$, and $X_{n}$ will be measurable with respect to the completion of $P$.

The converse is also known [3]: if the Strong Law holds, then $X_{1}$ almost surely equals an $L^{1}$-function, and is measurable with respect to the completion of $P$.

Can (1) be improved on? For instance, can the first or last almost sure inequality sometimes be replaced by an equality? Or can we say that in the nontrivial case it is almost surely true that $S_{n} / n$ diverges? Our main result shows that the answers to these questions are negative, and that the failure of the Strong Law for nonmeasurable $X_{1}$ is radical.

Theorem 1.3. Let $X_{1}, X_{2}, \ldots$ be iidpnmrvs with $E^{*}\left[\left|X_{1}\right|\right]<\infty$. Suppose $A$ is a nonempty proper subset of $\left[E_{*}\left[X_{1}\right], E^{*}\left[X_{1}\right]\right]$. Then each of the following is maximally nonmeasurable:

(i) the subset of $\Omega$ where $\liminf _{n \rightarrow \infty} S_{n}(n) / n$ is in $A$,

(ii) the subset of $\Omega$ where $\limsup _{n \rightarrow \infty} S_{n}(n) / n$ is in $A$,

(iii) the subset of $\Omega$ where $\lim _{n \rightarrow \infty} S_{n}(n) / n$ exists and is in $A$,

(iv) the subset of $\Omega$ where $\lim _{n \rightarrow \infty} S_{n}(n) / n$ exists,

(v) the subset of $\Omega$ where all the limit points of $S_{n}(n) / n$ are in $A$.

Thus in the nontrivial case (that is, when $\left[E_{*}\left[X_{1}\right], E^{*}\left[X_{1}\right]\right]$ has a nonempty proper subset, i.e., when $\left.E_{*}\left[X_{1}\right]<E^{*}\left[X_{1}\right]\right)$ nothing can be probabilistically said, with respect to $P$, about the limit points of $S_{n}(n) / n$ except that all the limit points lie within $\left[E_{*}\left[X_{1}\right], E^{*}\left[X_{1}\right]\right]$.

For completeness, here is a somewhat analogous result about the Weak Law:

TheOREM 1.4. Let $X_{1}, X_{2}, \ldots$ be iidpnmrvs with $E^{*}\left[\left|X_{1}\right|\right]<\infty$. Suppose $a \in\left[E\left[\left(X_{1}\right)_{*}\right], E\left[X_{1}^{*}\right]\right]$ and $\varepsilon>0$ is sufficiently small that $\left[E_{*}\left[X_{1}\right], E^{*}\left[X_{1}\right]\right]$ is not a subset of $[a-\varepsilon, a+\varepsilon]$. Then $P_{*}\left(\left|S_{n} / n-a\right|>\varepsilon\right) \rightarrow 0$ and $P^{*}\left(\left|S_{n} / n-a\right|>\varepsilon\right)$ $\rightarrow 1$ as $n \rightarrow \infty$.

The proof of both theorems will be based on the following easy fact about the existence of extensions of measures.

LEMMA 1.5. Suppose $f$ is a function on a probability space $(\Omega, \mathcal{F}, P)$ and $f$ is simple, i.e., takes on only finitely many values. Then there are 
extensions $P_{*}$ and $P^{*}$ of $P$ defined on the $\sigma$-field generated by $\mathcal{F}$ and $f$ such that $f=f_{*}$ almost surely with respect to $P_{*}$ and $f=f^{*}$ almost surely with respect to $P^{*}$.

It suffices to see this where $f$ is an indicator function, and in that case the result follows from the observation that if $A \subseteq \Omega$ and $\alpha \in\left[P_{*}(A), P^{*}(A)\right]$, then there is an extension $P_{\alpha}$ of $P$ to $\sigma\left(\mathcal{F} \cup\{A\}\right.$ ) such that $P_{\alpha}(A)=\alpha$ (cf. [2, p. 71]).

REMARK. Our proofs of the theorems would be much simpler if we could have this lemma for $f$ taking on infinitely many values, but alas the lemma is false in that case. To see the falsity, let $\Omega$ be the open square $(0,1)^{2}$, take $\mathcal{F}$ to be the $\sigma$-field of subsets of the form $A \times(0,1)$ for $A \subseteq(0,1)$ Lebesguemeasurable, take $P$ to be the restriction of Lebesgue measure to $\mathcal{F}$, and set $f(x, y)=y$. Then $f_{*}=0$ almost surely with respect to $P$ but there is no extension of $P$ with respect to which $f=0$ almost surely, since $f$ is nowhere equal to zero.

2. Proofs. We need some very easy preliminaries.

Lemma 2.1. Suppose that $f=g$ on a measurable set $B$. Then $f_{*}=g_{*}$ and $f^{*}=g^{*}$ almost surely on $B$.

Proof. Let $h(\omega)=f^{*}(\omega)$ for $\omega \notin B$ and let $h(\omega)=\min \left(f^{*}(\omega), g^{*}(\omega)\right)$ otherwise. Then $h$ is a measurable function such that $h \geq f$. Hence $h \geq f^{*}$ almost surely. So $g^{*} \geq h \geq f^{*}$ almost surely on $B$. In the same way, we see that $f^{*} \geq g^{*}$ almost surely on $B$. That $f_{*}=g_{*}$ almost surely on $B$ is proved the same way.

The following is a simple consequence of [6, Lemma 1.2.2].

Lemma 2.2. If $|f-g| \leq \varepsilon$ everywhere, then $\left|f_{*}-g_{*}\right| \leq \varepsilon$ and $\left|f^{*}-g^{*}\right| \leq \varepsilon$ almost surely.

The following trivial lemma encapsulates the strategy for the proof of our theorems.

Lemma 2.3. Suppose that $B$ is a subset of a probability space $(\Omega, \mathcal{F}, P)$ such that there are extensions $P_{1}$ and $P_{2}$ of $P$ so that $B$ is $P_{1}$ - and $P_{2}$ measurable with $P_{1}(B)=x_{1}$ and $P_{2}(B)=x_{2}$. Then $P_{*}(B) \leq x_{1}$ and $x_{2} \leq$ $P^{*}(B)$. In particular, if $P_{1}(B)=0$ and $P_{2}(B)=1$, then $B$ is maximally nonmeasurable.

Proof. We have $P_{*}(B)=P\left(B_{*}\right)=P_{1}\left(B_{*}\right) \leq P_{1}(B)=x_{1}$ and $P^{*}(B)=$ $P\left(B^{*}\right)=P_{2}\left(B^{*}\right) \geq P_{2}(B)=x_{2}$, where $B_{*}$ and $B^{*}$ are defined with respect to $P$. 
By Lemma 2.3, we need to show that for each of the subsets of $\Omega$ mentioned in Theorem 1.3 , there is an extension of $P$ that assigns measure zero to the subset and another that assigns to it measure one.

Finally, as we will soon see, the following lemma will yield all the results of Theorem 1.3 .

Lemma 2.4. Suppose $X_{1}, X_{2}, \ldots$ is a sequence of iidpnmrvs such that $E\left[\left|X_{1}\right|^{*}\right]<\infty$. Then for any $\alpha \in\left[E_{*}\left[X_{1}\right], E^{*}\left[X_{1}\right]\right]$ there is an extension $P^{\prime}$ of $P$ such that $P^{\prime}$-almost surely $S_{n} / n$ converges to $\alpha$. If $E_{*}\left[X_{1}\right]<E^{*}\left[X_{1}\right]$ then there is an extension $P^{\prime \prime}$ of $P$ such that $P^{\prime \prime}$-almost surely $S_{n}$ diverges.

Proof. Write $E_{P}[f]$ for the expectation of $f$ with respect to $P$, i.e., $\int_{\Omega} f(\omega) d P(\omega)$. The variables $X_{1}, X_{2}, \ldots$ are defined by $X_{n}\left(\omega_{1}, \omega_{2}, \ldots\right)=$ $\Psi\left(\omega_{n}\right)$ on our product space $\Omega$ for some real-valued function $\Psi$.

Let $X_{n}^{\prime}=X_{n} \cdot 1_{\left\{\left|X_{n}\right| \leq n\right\}}$.

Let $A_{n, \pm}=\left\{ \pm X_{n}>n\right\}$. Let $A_{n}=\left\{\left|X_{n}\right|>n\right\}=A_{n,+} \cup A_{n,-}$. Observe that $A_{n,+} \subseteq\left\{X_{n}^{*}>n\right\}$ and $A_{n,-} \subseteq\left\{\left(X_{n}\right)_{*}<-n\right\}$. Let $B_{n}=\left\{\left|X_{n}^{*}\right|>n\right\} \cup$ $\left\{\left|\left(X_{n}\right)_{*}\right|>n\right\}$. Clearly $A_{n} \subseteq B_{n}$. Then

$$
\sum_{n=1}^{\infty} P\left(B_{n}\right) \leq \sum_{n=1}^{\infty} P\left(\left|\left(X_{n}\right)_{*}\right|>n\right)+\sum_{n=1}^{\infty} P\left(\left|X_{n}^{*}\right|>n\right)<\infty
$$

since $\sum_{n=1}^{\infty} P(|X|>n) \leq \int_{0}^{\infty} P(|X|>t) d t=E[|X|]$, and since both $\left(X_{n}\right)_{*}$ and $X_{n}^{*}$ have finite expectations given that $\left|X_{n}\right|^{*}$ does. In particular, almost surely, only finitely many of the $B_{n}$ occur by Borel-Cantelli. Since $A_{n} \subseteq B_{n}$, almost surely only finitely many of the $A_{n}$ occur.

We now claim that $E\left[\left(X_{n}^{\prime}\right)_{*}\right] \rightarrow E\left[\left(X_{1}\right)_{*}\right]$ and $E\left[\left(X_{n}^{\prime}\right)^{*}\right] \rightarrow E\left[X_{1}^{*}\right]$ as $n \rightarrow \infty$. We only need to prove the latter convergence since the former follows by applying the latter to the iidpnmrv sequence $\left\{-X_{n}\right\}$. Now, outside of $B_{n}$, we have $X_{n}^{\prime}=X_{n}$, and since $B_{n}$ is measurable it follows from Lemma 2.1 that outside of $B_{n}$ we have $\left(X_{n}^{\prime}\right)^{*}=X_{n}^{*}$ almost surely. Moreover, everywhere on $B_{n}$ we have $X_{n}^{\prime}=0$ and hence $\left(X_{n}^{\prime}\right)^{*}=0$ by Lemma 2.1. Thus

$$
\begin{aligned}
\left|E\left[\left(X_{n}^{\prime}\right)^{*}\right]-E\left[X_{1}^{*}\right]\right| & =\left|E\left[\left(X_{n}^{\prime}\right)^{*}\right]-E\left[X_{n}^{*}\right]\right| \leq E\left[\left|\left(X_{n}^{\prime}\right)^{*}-X_{n}^{*}\right|\right] \\
& \leq E\left[\left|\left(X_{n}^{\prime}\right)^{*}-X_{n}^{*}\right| \cdot 1_{B_{n}}\right]=E\left[\left|X_{n}^{*}\right| \cdot 1_{B_{n}}\right] \\
& \leq E\left[\left|X_{n}^{*}\right| \cdot 1_{\left\{\left|X_{n}\right|^{*}>n\right\}}\right]=E\left[\left|X_{1}^{*}\right| \cdot 1_{\left\{\left|X_{1}\right|^{*}>n\right\}}\right] \rightarrow 0,
\end{aligned}
$$

since $E\left[\left|X_{1}\right|^{*}\right]<\infty$.

Let $\Psi_{n}^{\prime}=\Psi \cdot 1_{\{|\Psi| \leq n\}}$ so that $X_{n}^{\prime}\left(\omega_{1}, \omega_{2}, \ldots\right)=\Psi_{n}^{\prime}\left(\omega_{n}\right)$. Choose a simple function $\Psi_{1, n}$ such that both $\left|\Psi_{1, n}-\Psi_{n}^{\prime}\right| \leq 1 / n$ and $\left|\Psi_{1, n}\right| \leq n$ everywhere on $\Omega_{1}$.

By Lemma 1.5 there is an extension $P_{1, n, 0}$ of $P_{1}$ such that $\Psi_{1, n}$ is $P_{1, n, 0^{-}}$ measurable and $P_{1, n, 0}$-almost surely $\Psi_{1, n}=\left(\Psi_{1, n}\right)_{*}$, and an extension $P_{1, n, 1}$ of $P_{1}$ such that $\Psi_{1, n}$ is $P_{1, n, 1}$-measurable and $P_{1, n, 1}$-almost surely $\Psi_{1, n}=\Psi_{1, n}^{*}$. Let $\mathcal{F}_{1, n}$ be the $\sigma$-field on $\Omega_{1}$ generated by $\mathcal{F}_{1}$ and $\Psi_{1, n}$. 
For $i=0,1$, let $P^{i}$ be the product of the measures $P_{1,1, i}, P_{1,2, i}, P_{1,3, i}, \ldots$ This is an extension of $P$.

Let $Y_{n}\left(\omega_{1}, \omega_{2}, \ldots\right)=\Psi_{1, n}\left(\omega_{n}\right)$. By Proposition 1.2, $\left(Y_{n}\right)_{*}\left(\omega_{1}, \omega_{2}, \ldots\right)=$ $\left(\Psi_{1, n}\right)_{*}\left(\omega_{n}\right)$ for $P$-almost all $\left(\omega_{1}, \omega_{2}, \ldots\right)$. Then $P^{0}$-almost surely we have $Y_{n}=\left(Y_{n}\right)_{*}$ and $P^{1}$-almost surely $Y_{n}=Y_{n}^{*}$ (where $\left(Y_{n}\right)_{*}$ and $Y_{n}^{*}$ are defined with respect to $P)$. Define the measure $P^{\prime}=(1-a) P^{0}+a P^{1}$, where $a$ is such that $\alpha=(1-a) E_{P}\left[\left(X_{1}\right)_{*}\right]+a E_{P}\left[X_{1}^{*}\right]$. Then $E_{P^{\prime}}\left[Y_{n}\right]=$ $(1-a) E_{P}\left[\left(Y_{n}\right)_{*}\right]+a E_{P}\left[Y_{n}^{*}\right]$. Now $\left|\left(Y_{n}\right)_{*}-\left(X_{n}^{\prime}\right)_{*}\right| \leq 1 / n$ and $\left|Y_{n}^{*}-\left(X_{n}^{\prime}\right)^{*}\right| \leq$ $1 / n$ everywhere by Lemma 2.2 and the choice of $\Psi_{1, n}$, so by uniform convergence we have $E\left[\left(Y_{n}\right)_{*}-\left(X_{n}^{\prime}\right)_{*}\right]$ and $E\left[Y_{n}^{*}-\left(X_{n}^{\prime}\right)^{*}\right]$ converging to zero. Moreover, we have already seen that $E\left[\left(X_{n}^{\prime}\right)_{*}\right] \rightarrow E\left[\left(X_{1}\right)_{*}\right]$ and $E\left[\left(X_{n}^{\prime}\right)^{*}\right] \rightarrow$ $E\left[X_{1}^{*}\right]$, so $E_{P}\left[\left(Y_{n}\right)_{*}\right] \rightarrow E_{P}\left[\left(X_{1}\right)_{*}\right]$ and $E_{P}\left[Y_{n}^{*}\right] \rightarrow E_{P}\left[X_{1}^{*}\right]$. Thus, $E_{P^{\prime}}\left[Y_{n}\right] \rightarrow$ $(1-a) E_{P}\left[\left(X_{1}\right)_{*}\right]+a E_{P}\left[X_{1}^{*}\right]=\alpha$.

Now $\left|Y_{n}-X_{n}^{\prime}\right| \leq 1 / n$ everywhere and $\left|X_{n}^{\prime}\right| \leq \max \left(\left|\left(X_{n}\right)_{*}\right|,\left|X_{n}^{*}\right|\right) \cdot 1_{B_{n}}$. Thus

$$
\begin{aligned}
\sum_{n=1}^{\infty} & \frac{\operatorname{Var}_{P^{\prime}}\left[Y_{n}\right]}{n^{2}} \leq \sum_{n=1}^{\infty} \frac{E\left[Y_{n}^{2}\right]}{n^{2}}=\sum_{n=1}^{\infty} \frac{E_{P^{\prime}}\left[\left(X_{n}^{\prime}+\left(Y_{n}-X_{n}^{\prime}\right)\right)^{2}\right]}{n^{2}} \\
& \leq 2 \sum_{n=1}^{\infty} \frac{E_{P^{\prime}}\left[\left(X_{n}^{\prime}\right)^{2}\right]}{n^{2}}+\sum_{n=1}^{\infty} \frac{2}{n^{3}} \\
& \leq 2 \sum_{n=1}^{\infty} \frac{E_{P^{\prime}}\left[\left(\left(X_{n}\right)_{*}\right)^{2} \cdot 1_{B_{n}}\right]}{n^{2}}+2 \sum_{n=1}^{\infty} \frac{E_{P^{\prime}}\left[\left(X_{n}^{*}\right)^{2} \cdot 1_{B_{n}}\right]}{n^{2}}+\sum_{n=1}^{\infty} \frac{2}{n^{3}} \\
& =2 \sum_{n=1}^{\infty} \frac{E_{P}\left[\left(\left(X_{n}\right)_{*}\right)^{2} \cdot 1_{B_{n}}\right]}{n^{2}}+2 \sum_{n=1}^{\infty} \frac{E_{P}\left[\left(X_{n}^{*}\right)^{2} \cdot 1_{B_{n}}\right]}{n^{2}}+O(1) \\
& \leq 2 \sum_{n=1}^{\infty} \frac{E_{P}\left[\left(\left(X_{n}\right)_{*}\right)^{2} \cdot 1_{\left\{\left|\left(X_{n}\right)_{*}\right|>n\right\}}\right]}{n^{2}}+2 \sum_{n=1}^{\infty} \frac{E_{P}\left[\left(X_{n}^{*}\right)^{2} \cdot 1_{\left\{\left|X_{n}^{*}\right|>n\right\}}\right]}{n^{2}}+O(1),
\end{aligned}
$$

where the last equality follows from the fact that $P$ is an extension of $P^{\prime}$, and $\left(X_{n}\right)_{*}$ and $X_{n}^{*}$ are $P$-measurable. The finiteness of the right hand side is then a consequence of the proof of [1, Lemma 2.4.3]. It then follows from Kolmogorov's Strong Law [5, Theorem 5.8] that $P^{\prime}$-almost surely $\left(Y_{1}+\cdots+\right.$ $\left.Y_{n}-E\left[Y_{1}+\cdots+Y_{n}\right]\right) \rightarrow 0$ and hence $\left(Y_{1}+\cdots+Y_{n}\right) / n \rightarrow \alpha$.

Let $T_{n}=Y_{1}+\cdots+Y_{n}$. Let $S_{n}^{\prime}=X_{1}^{\prime}+\cdots+X_{n}^{\prime}$. Since $\left|X_{n}^{\prime}-Y_{n}\right| \leq 1 / n$ everywhere, $\left|S_{n}^{\prime} / n-T_{n} / n\right| \leq(1 / n)(1+1 / 2+\cdots+1 / n)$ and the right hand side converges to zero. Moreover, $P$-almost surely for all but finitely many $n$ we have $X_{n}=X_{n}^{\prime}$, so that $P$-almost surely $\left(S_{n}-S_{n}^{\prime}\right) / n \rightarrow 0$. Thus, $P$-almost surely we have $\left(S_{n}-T_{n}\right) / n \rightarrow 0$. But $P^{\prime}$ extends $P$, so this also holds $P^{\prime}$-almost surely. But since $P^{\prime}$-almost surely $T_{n} / n \rightarrow \alpha$, we see that $S_{n} / n$ converges $P^{\prime}$-almost surely to $\alpha$. 
To construct $P^{\prime \prime}$, first recall that $E_{P}\left[\left(Y_{n}\right)_{*}\right] \rightarrow E_{P}\left[\left(X_{1}\right)_{*}\right]$ and $E_{P}\left[Y_{n}^{*}\right] \rightarrow$ $E_{P}\left[X_{1}^{*}\right]$. Let $\gamma=E_{P}\left[\left(X_{1}\right)_{*}\right]$ and $\delta=E_{P}\left[X_{1}^{*}\right]$. For $n>0$, let $a_{n}$ be a strictly increasing sequence of positive integers such that (a) $\mid E_{P}\left[\left(Y_{k}\right)_{*}\right]$ $E_{P}\left[\left(X_{1}\right)_{*}\right] \mid<1 / n$ and $\left|E_{P}\left[Y_{k}^{*}\right]-E_{P}\left[X_{1}^{*}\right]\right|<1 / n$ for all $k \geq a_{n}$, and (b) $a_{n+1} / a_{n} \rightarrow \infty$. Let $a_{0}=1$.

Let $L_{n}=\left\{a_{n-1}+1, a_{n-1}+2, \ldots, a_{n}\right\}$. Let $L=L_{1} \cup L_{3} \cup L_{5} \cup \cdots$. For $n \in L$, let $\alpha_{n}=E_{P}\left[\left(Y_{n}\right)_{*}\right]$, and for $n \notin L$, let $\alpha_{n}=E_{P}\left[Y_{n}^{*}\right]$. Let $\beta_{n}=\alpha_{1}+\cdots+\alpha_{n}$. We now claim that $\beta_{a_{2 n}} / a_{2 n} \rightarrow \delta$ and $\beta_{a_{2 n+1}} / a_{2 n+1} \rightarrow \gamma$ as $n \rightarrow \infty$. For, since both $E_{P}\left[\left(Y_{n}\right)_{*}\right]$ and $E_{P}\left[Y_{n}^{*}\right]$ converge, there is an $M<\infty$ such that $\left|\alpha_{k}\right| \leq M$ for all $K$, and then by the choice of $a_{n}$,

$$
\begin{aligned}
\beta_{a_{2 n}}-a_{2 n} \delta & =\sum_{k=a_{2 n-1}+1}^{a_{2 n}}\left(E_{P}\left[Y_{k}^{*}\right]-\delta\right)-a_{2 n-1} \delta+\sum_{k=1}^{a_{2 n-1}} \alpha_{k} \\
& =O\left(a_{2 n} /(2 n-1)\right)+O\left(a_{2 n-1}\right) .
\end{aligned}
$$

Since $a_{2 n-1} / a_{2 n} \rightarrow 0$ by condition (b) above, we see that $\beta_{a_{2 n}} / a_{2 n}-\delta$ converges to 0 as desired. Likewise,

$$
\begin{aligned}
\beta_{a_{2 n+1}}-a_{2 n+1} \gamma & =\sum_{k=a_{2 n}+1}^{a_{2 n+1}}\left(E_{P}\left[\left(Y_{k}\right)_{*}\right]-\gamma\right)-a_{2 n} \gamma+\sum_{k=1}^{a_{2 n}} \alpha_{k} \\
& =O\left(a_{2 n+1} / 2 n\right)+O\left(a_{2 n}\right),
\end{aligned}
$$

and so $\beta_{a_{2 n+1}} / a_{2 n+1} \rightarrow \gamma$.

Now define $P_{1, n}^{\prime \prime}$ to be equal to $P_{1, n, 0}$ if $n \in L$, and to $P_{1, n, 1}$ if $n \notin L$. Let $P^{\prime \prime}$ be the product of the measures $P_{1,1}^{\prime \prime}, P_{1,2}^{\prime \prime}, \ldots$

In exactly the same way as we proved above using Kolmogorov's Strong Law that $P^{\prime}$-almost surely $\left(T_{n}-E_{P^{\prime}}\left[T_{n}\right]\right) / n \rightarrow 0$, we can also show that $P^{\prime \prime}$ almost surely $\left(T_{n}-E_{P^{\prime \prime}}\left[T_{n}\right]\right) / n \rightarrow 0$. But $E_{P^{\prime \prime}}\left[T_{n}\right]=\beta_{n}$, since $E_{P^{\prime \prime}}\left[Y_{n}\right]=$ $E_{P}\left[\left(Y_{n}\right)_{*}\right]$ if $n \in L$ and $E_{P^{\prime \prime}}\left[Y_{n}\right]=E_{P}\left[Y_{n}^{*}\right]$ otherwise. Thus, $P^{\prime \prime}$-almost surely $T_{a_{2 n}} / a_{2 n} \rightarrow \delta$ and $T_{a_{2 n+1}} / a_{2 n+1} \rightarrow \gamma$.

But we have already seen that $\left(S_{n} / n-T_{n}\right) / n$ converges to zero $P$-almost surely, and hence also $P^{\prime \prime}$-almost surely. Thus $P^{\prime \prime}$-almost surely $S_{a_{2 n}} / a_{2 n} \rightarrow \delta$ and $S_{a_{2 n+1}} / a_{2 n+1} \rightarrow \gamma$. Since $\delta>\gamma$, our desired divergence result follows.

Proof of Theorem 1.3. Choose any $a_{1} \in A$. By Lemma 2.4, we have an extension $P^{\prime}$ of $P$ such that $P^{\prime}$-almost surely $S_{n} / n$ converges to $a_{1}$. Moreover, since $A$ is a proper nonempty subset of $\left[E_{P}\left[\left(X_{1}\right)_{*}\right], E_{P}\left[X_{1}^{*}\right]\right]$, this interval must contain at least two points and hence $E_{P}\left[\left(X_{1}\right)_{*}\right]<E_{P}\left[X_{1}^{*}\right]$, so by the same lemma there is an extension $P^{\prime \prime}$ of $P$ such that $P^{\prime \prime}$-almost surely $S_{n} / n$ diverges. Now choose any $a_{2} \in\left[E_{P}\left[\left(X_{1}\right)_{*}\right], E_{P}\left[X_{1}^{*}\right]\right]-A$. Again by Lemma 2.4 there is an extension $P^{\prime \prime \prime}$ of $P$ such that $P^{\prime \prime \prime}$-almost surely $S_{n} / n$ converges to $a_{2}$.

All the events described in (i)-(v) will happen whenever $S_{n} / n \rightarrow a_{1}$, and so they all have $P^{\prime}$-measure 1 . Events (i), (ii), (iii) and (v) cannot happen 
when $S_{n} / n \rightarrow a_{2}$, and so they all have $P^{\prime \prime \prime}$-measure 0 . And event (iv) has $P^{\prime \prime}$-measure 0 . Thus, each event has measure 1 under one extension of $P$ and measure 0 under some other extension, and so by Lemma 2.3, each event is maximally $P$-nonmeasurable.

Proof of Theorem 1.4. Let $a$ and $\varepsilon$ be as in the statement of the theorem. The conditions of the theorem guarantee that there is a point $b \in$ $\left[E_{*}\left[X_{1}\right], E^{*}\left[X_{1}\right]\right]-[a-\varepsilon, a+\varepsilon]$. Let $\gamma=|b-a|-\varepsilon$. Since $b \notin[a-\varepsilon, a+\varepsilon]$, we have $\gamma>0$.

By Lemma 2.4. let $P^{\prime}$ be an extension of $P$ such that $P^{\prime}$-almost surely $S_{n} / n$ converges to $a$, and let $P^{\prime \prime}$ be an extension of $P$ such that $P^{\prime \prime}$-almost surely $S_{n} / n$ converges to $b$. Then $S_{n} / n$ converges to $a$ in $P^{\prime}$-probability and to $b$ in $P^{\prime \prime}$-probability. Hence $\lim _{n \rightarrow \infty} P^{\prime}\left(\left|S_{n} / n-a\right|>\varepsilon\right)=0$. By Lemma 2.3, we have $\lim _{n \rightarrow \infty} P_{*}\left(\left|S_{n} / n-a\right|>\varepsilon\right)=0$. Moreover, $0=$ $\lim _{n \rightarrow \infty} P^{\prime \prime}\left(\left|S_{n} / n-b\right|>\gamma\right) \geq \lim \sup _{n \rightarrow \infty} P^{\prime \prime}\left(\left|S_{n} / n-a\right| \leq \varepsilon\right)$ by the choice of $\gamma$. Thus $P^{\prime \prime}\left(\left|S_{n} / n-a\right| \leq \varepsilon\right)$ converges to 0 , and so $P^{\prime \prime}\left(\left|S_{n} / n-a\right|>\varepsilon\right)$ converges to 1 , hence $P^{*}\left(\left|S_{n} / n-a\right|>\varepsilon\right)$ also converges to 1 .

Acknowledgments. The author wishes to thank Arthur Paul Pedersen for several discussions, and an anonymous referee for a number of helpful suggestions.

\section{References}

[1] R. Durrett, Probability: Theory and Examples, 4th ed., Cambridge Univ. Press, Cambridge, 2010.

[2] P. R. Halmos, Measure Theory, Springer, New York, 1974.

[3] J. Hoffmann-Jørgensen, The law of large numbers for nonmeasurable and nonseparable random elements, Astérisque 131 (1985), 299-356.

[4] J. Hoffmann-Jørgensen, Perfect independence and stochastic inequalities, MaPhySto, Research Report, Dept. Math. Sci., Univ. of Aarhus, 2002, http://www.maphysto.dk/ cgi-bin/gp.cgi?publ=375.

[5] J. N. McDonald and N. A. Weiss, A Course in Real Analysis, Academic Press, San Diego, 1999.

[6] A. W. van der Vaart and J. A. Wellner, Weak Convergence and Empirical Processes. With Applications to Statistics, Springer, New York, 1996.

Alexander R. Pruss

Department of Philosophy

Baylor University

One Bear Place \#97273

Waco, TX 76798-7273, U.S.A.

E-mail: alexander_pruss@baylor.edu 